\title{
Efeito do espaçamento e arranjo de plantio na relação hipsométrica de eucalipto em sistema consorciado de produção
}

\section{Clebson Lima CERQUEIRA ${ }^{1 *}$, Rômulo MÔRA ${ }^{2}$, Helio TONINI ${ }^{3}$, Diogo Guido Streck VENDRUSCOLO ${ }^{4}$, Luciano Rodrigo LANSSANOVA ${ }^{5}$, Julio Eduardo ARCE ${ }^{1}$, Carlos Cezar Cavassin DINIZ ${ }^{1}$}

\author{
${ }^{1}$ Programa de Pós-Graduação em Engenharia Florestal, Universidade Federal do Paraná, Curitiba, PR, Brasil. \\ ${ }^{2}$ Programa de Pós-Graduação em Ciências Florestais e Ambientais, Universidade Federal de Mato Grosso, Cuiabá, PR, Brasil. \\ ${ }^{3}$ Embrapa Pecuária Sul, Bagé, RS, Brasil. \\ ${ }^{4}$ Proterra-Projetos, Agrimesura \& Ambiental, Alto Boa Vista, MT, Brasil. \\ ${ }^{5}$ Instituto Federal de Ciência, Tecnologia e Educação de Mato Grosso, Juína, MT, Brasil. \\ *E-mail: clebson.mensuracao@gmail.com
}

Recebido em dezembro/2018; Aceito em junho/2019.

RESUMO: Dada a importância dos sistemas de integração Lavoura-Pecuária-Floresta (iLPF), tornam-se indispensáveis a realização de pesquisas sobre a modelagem da altura para dar suporte na gestão e quantificação dos recursos disponíveis. Neste sentido, objetivou-se avaliar o efeito do espaçamento e arranjos de plantio na relação hipsométrica de um híbrido Eucalyptus grandis $x$ Eucalyptus urophylla em iLPF, utilizando modelos tradicionais para estimativa da altura comercial e total. Os dados foram obtidos em sistema de (iLPF), em áreas experimentais implantadas pela Empresa Brasileira de Pesquisa Agropecuária (Embrapa Agrossilvipastoril), localizadas no município de Sinop, região norte de Mato Grosso. Para estimativa da altura comercial e total foram testados os modelos de Stoffels, Curtis, Hiperbólico, Henricksen e Assmann, com a precisão avaliada por meio de indicadores estatísticos e análise gráfica de resíduos. A influência do espaçamento e arranjo de plantio na relação hipsométrica foi avaliada por meio do teste de identidade de modelos de Graybill. Com base nas estatísticas e análise gráfica o modelo de Assmann apresentou melhor acurácia de ajuste para estimativa da altura total. Para estimativa da altura comercial o modelo Hiperbólico obteve melhor desempenho. $\mathrm{O}$ teste de identidade revelou que o espaçamento e arranjo de plantio influenciam de forma significativa na relação hipsométrica e uma única equação não deverá ser ajustada para representar a altura comercial e total nos diferentes tratamentos em iLPF.

Palavras-chave: modelagem; teste de identidade; altura das árvores.

\section{Effect of spacing and planting arrangement on the hypsometric relation of eucalyptus in consortium production systems}

\begin{abstract}
Given the importance of crop-livestock-forest integration systems (iLPF), it is essential to carry out research on height modeling to support the management and quantification of available resources. In this study, we aimed to adjust traditional models of hypsometric relation and to evaluate the effect of spacing and planting arrangements in the equations adjustment. The data were obtained from the eucalyptus trees in an integrated-crop-livestock-forest system (iCLF), in experimental areas implemented by the Brazilian Agricultural Research Corporation (Embrapa Agrossilvipastoril), located in the municipality of Sinop, northern region of Mato Grosso, Brazil. The precision and accuracy of the hypsometric models was evaluated through statistical indicators and graphic residue analysis. The influence of spacing and planting arrangement on the hypsometric relation was performed through the model identity test of Gaybiil (2000). Based on the statistics and graphical analysis the Assmann model presented better accuracy of fit for total height estimation. To estimate the commercial height, the Hyperbolic model obtained better performance. The identity test revealed that plant spacing, and arrangement influence significantly the hypsometric relationship and a single equation should not be adjusted to represent the total and commercial height in the different treatments in iCLF system.
\end{abstract}

Keywords: modeling; identity test; tree height.

\section{INTRODUÇÃO}

A integração Lavoura-Pecuária-Floresta (iLPF), que une diferentes sistemas de produção na mesma área, vem contribuindo de forma significativa para a intensificação do uso da terra, com ganhos ambientais e diversificação das receitas do produtor. Segundo a Indústria Brasileira de Árvores (2016), no ano de 2015 o Brasil possuía uma área ocupada de 2 milhões de hectares em diferentes formatos da estrutura iLPF.

O gênero Eucalyptus, apresenta rápido crescimento, e tem sido bastante utilizado para compor sistemas de iLPF (MACEDO et al., 2010). Nesses modelos de Sistemas Agroflorestais, culturas agrícolas, árvores, pastagens e animais são integrados visando potencializar interações ecológicas e 
econômicas (TORRES et al., 2016). Neste cenário, pesquisas sobre silvicultura e manejo florestal em sistemas de iLPF, tornam-se indispensáveis para gestão e quantificação dos recursos disponíveis.

A modelagem da altura em povoamentos de eucalipto estabelecidos em monocultivo é um assunto bastante difundido no setor florestal. Entretanto, em se tratando de sistemas iLPF, onde são estabelecidos diferentes arranjos de plantio e espaçamentos, ainda são escassos os estudos de modelagem para estimativa de variáveis dendrométricas, sendo encontrados apenas alguns estudos para estimativa do volume, crescimento, afilamento (MÜLLER et al., 2011), produção (SALLES et al., 2012; TORRES et al., 2016), distribuição diamétrica (BINOTI et al., 2012) e altura (SOUZA et al., 2016).

A variável altura pode ser obtida por medição direta em árvores de pequeno porte ou abatida, bem como pelo método indireto, empregando hipsômetros ou clinômetros (CAMPOS; LEITE, 2013). Contudo, devido às limitações operacionais e econômicas em se medir todas as alturas, são empregadas as equações hipsométricas para estimativa das alturas nos inventários florestais (SCOLFORO, 2006; MENDONÇA et al., 2015).

A relação hipsométrica sofre influência de diversos fatores ambientais e do povoamento, como o sítio, idade, material genético, densidade, tamanho da copa, arranjo espacial e tratamentos silviculturais em geral (BARTOSZECK et al., 2004). Entretanto, recomendam-se ajustes nos modelos para cada condição específica de acordo com a região ou condições de cultivo dos povoamentos florestais (CURTIS, 1967; SCOLFORO, 2005). Bartoszeck et al. (2002), acrescentam que os modelos hipsométricos que consideram esses fatores são empregados com menos frequência por serem mais complexos e de difícil ajuste, sendo preferido o uso de equações tradicionais disponíveis na literatura florestal.

Segundo Vendruscolo et al. (2015), em situações onde os povoamentos florestais são implantados ou conduzidos sob diferentes tratamentos, os ajustes de modelos hipsométricos devem ser realizados para cada situação, o que pode acarretar em um número elevado de equações. Dessa maneira, é importante testar formas de agrupamento entre os diferentes tratamentos por meio de procedimentos estatísticos, sendo usualmente realizados testes de identidade de modelos (ARAÚJO et al., 2012; MORAES NETO et al., 2012; VENDRUSCOLO et al., 2015).

Partindo-se da hipótese de que os diferentes arranjos de iLPF influenciam na relação hipsométrica, objetivou-se avaliar o efeito do espaçamento e arranjos de plantio utilizando modelos tradicionais para estimativa da altura comercial e total de um clone do híbrido Eucalyptus grandis $x$ Eucalyptus urophylla em iLPF.

\section{MATERIAL E MÉTODOS}

\subsection{Caracterização da área de estudo}

Os dados para realização do estudo foram obtidos em sistema de integração Lavoura-Pecuária-Floresta (iLPF), em duas áreas experimentais implantadas pela Empresa Brasileira de Pesquisa Agropecuária (Embrapa Agrossilvipastoril), denominadas iLPF leite e iLPF corte, localizadas no município de Sinop, na região norte de Mato Grosso.

De acordo com a classificação de Köppen, o clima da região é do tipo tropical de monção, com três meses de seca e precipitação pluviométrica acumulada no ano entre 2.500 e
$2.800 \mathrm{~mm}$, temperatura média anual aproximada de 24 a 26 ${ }^{\circ} \mathrm{C}$, temperaturas máximas e mínimas próximas a 40 e $20{ }^{\circ} \mathrm{C}$, respectivamente, e altitude de $380 \mathrm{~m}$ (ALVARES et al., 2013). Além de relevo plano e predomínio do Latossolo VermelhoAmarelo Argiloso (SEMA-MT, 2014).

A área experimental foi dividida em dois experimentos com mesmas características de solo e relevo, implantados em delineamento de blocos casualizados (DBC) com quatro tratamentos: árvores periféricas na pastagem (T1), lavourapecuária-floresta (T2), pecuária-floresta (T3) e plantio homogêneo (T4).

No iLPF leite foi instalado o tratamento T1, com arranjo das árvores em renques duplos e $\mathrm{T} 2$ as árvores foram estabelecidas em faixas com três linhas de plantio, consorciados (T1 e T2) com pastagem de capim-piatã (Brachiaria brizantha cv. Piatã) em integração com milho para silagem e criação de gado leiteiro girolanda. No iLPF corte foi instalado o tratamento $\mathrm{T} 3 \mathrm{com}$ as árvores estabelecidas em faixas com três linhas de plantio, consorciados com capimpiatã (Brachiaria brizantha cv. Piatã) e criação de boi nelore para corte. O T4 foi composto apenas pelo plantio homogêneo do componente florestal.

Os tratamentos foram estabelecidos em diferentes arranjos de integração (Tabela 1). O componente florestal utilizado foi um clone do híbrido Eucalyptus grandis $x$ Eucalyptus urophylla e a orientação de plantio das árvores nas linhas foi no sentido leste oeste.

Tabela 1. Árvores mensuradas nos diferentes tratamentos do sistema de integração Lavoura-Pecuária-Floresta.

Table 1. Trees measured in different treatments integrated-croplivestock-forest system.

\begin{tabular}{ccccc}
\hline Tratamentos & Arranjo de plantio $(\mathrm{m})$ & ${\text { Arv } / \mathrm{ha}^{-1}}^{-1}$ & $\mathrm{~N}$ & $\mathrm{~L}$ \\
\hline $\mathrm{T} 1$ & $3 \times 2 \times 52$ & 189 & 21 & 2 \\
$\mathrm{~T} 2$ & $3 \times 2 \times 15$ & 714 & 56 & 3 \\
$\mathrm{~T} 3$ & $3,5 \times 3 \times 30$ & 270 & 54 & 3 \\
T4 & $3,5 \times 3$ & 952 & 55 & - \\
\hline Total & - & & 186 \\
\hline
\end{tabular}

$\mathrm{N}=$ número de árvores mensuradas, $\mathrm{Arv} / \mathrm{ha}^{-1}=$ número de árvores por hectare; $\mathrm{L}=$ número de linhas de plantio das árvores nos tratamentos (T1, T2, T3).

\subsection{Base de dados}

Os dados para realização deste trabalho foram obtidos por meio de inventário florestal, sendo mensurados o diâmetro com casca a 1,30 m de altura (d), a altura total (ht) e altura comercial (hc) definida até o primeiro galho vivo com auxílio do hipsômetro Haglöf, perfazendo um total de 186 árvores aos 51 meses de idade.

Para avaliar o efeito do espaçamento e arranjo de plantio nas variáveis dendrométricas (d, ht e hc) das árvores, empregou-se a análise de variância (ANOVA) ao nível de 5\% de significância. Caso constatado diferença estatística pela ANOVA, aplicou-se o teste de Tukey.

Para estudar a relação hipsométrica foram testados cinco modelos estatísticos, sendo quatro lineares e um não linear (Tabela 2). Os modelos lineares foram ajustados pelo método dos mínimos quadrados ordinários e o não linear pelo método interativo do algoritmo de Gauss-Newton.

A precisão e acurácia das estimativas foram avaliadas com base nos seguintes critérios estatísticos de ajuste: coeficiente de determinação ajustado $\left(\mathrm{R}_{\text {aj. }}^{2}\right)$, erro padrão da estimativa em porcentagem $\left(\mathrm{S}_{\mathrm{yx} \%}\right)$, significância dos coeficientes de regressão $(\beta)$ e análise gráfica dos resíduos $(\mathrm{E} \%)$, calculados pelas equações, 1,2 e 3 . 
Tabela 2. Modelos de relação hipsométrica testados para estimativa da altura comercial e total das árvores do sistema de integração Lavoura-Pecuária-Floresta.

Table 2. Hypsometric relation models tested for tree height estimation in the crop-livestock-forest integration system .

\begin{tabular}{cc}
\hline Denominação & Modelo \\
\hline Stoffels & $\ln (\mathrm{h})=\beta_{0}+\beta_{1} \ln (\mathrm{d})+\varepsilon$ \\
Curtis & $\ln (\mathrm{h})=\beta_{0}+\beta_{1} \frac{1}{\mathrm{~d}}+\varepsilon$ \\
Hiperbólico & $\mathrm{h}=\beta_{0}+\frac{\beta_{1}}{\mathrm{~d}^{2}}+\varepsilon$ \\
Henricksen & $\mathrm{h}=\beta_{0}+\beta_{1} \ln (\mathrm{d})+\varepsilon$ \\
Assmann & $\mathrm{h}=\beta_{0}+\beta_{1} \frac{1}{\mathrm{~d}}+\varepsilon$
\end{tabular}

em que: $\ln =$ logaritmo natural; $\mathrm{h}=$ altura total, comercial $(\mathrm{m}) ; \mathrm{d}=$ diâmetro a $1,30 \mathrm{~m}$ de altura $(\mathrm{cm}) ; \beta_{0}$ e $\beta_{1}=$ coeficientes; $\varepsilon=$ erro aleatório.

$$
\begin{aligned}
& \mathrm{R}^{2}{ }_{\text {aj. }}=1-\left(\frac{S Q_{\text {res }}}{S Q_{\text {tot }}}\right) *\left(\frac{\mathrm{n}-1}{\mathrm{n}-\mathrm{p}}\right) * 100 \\
& S_{y x}=\sqrt{\frac{\sum_{i=1}^{n}\left(Y_{i}-\hat{Y}_{i}\right)^{2}}{n-p}} S_{y x} \%=\frac{S_{y x}}{\bar{Y}} * 100 \\
& \mathrm{E}_{\%}=\left(\frac{\mathrm{Y}_{\mathrm{i}}-\widehat{\mathrm{Y}}_{\mathrm{i}}}{\mathrm{Y}_{\mathrm{i}}}\right) * 100
\end{aligned}
$$

em que: $Y_{i}=$ variável observada; $\widehat{Y}_{i}=$ variável estimada; $\bar{Y}=$ média aritmética da variável; $\mathrm{n}=$ número de observações; $\mathrm{p}=$ número de coeficientes do modelo associados a variável independente; $S Q_{\text {res }}=$ soma dos quadrados dos resíduos; e $S Q_{t o t}=$ soma de quadrados total

Para modelos estatísticos em escala logarítmica, a discrepância logarítmica foi corrigida pelo Fator de Correção de Meyer (FM), (Equação 4), conforme Machado et al. (2008).

$$
\mathbf{F M}=\mathbf{e}^{0,5 * \mathrm{QM}_{\mathrm{res}}}
$$

em que: $e=$ Base do logaritmo natural (2,718281828...); $Q M_{\text {res }}=$ Quadrado médio dos resíduos.

\subsection{Teste de identidade de modelos}

A influência do espaçamento e arranjo de plantio na relação hipsométrica foi avaliada pelo teste de identidade de modelos proposto por Graybill (2000). O teste foi realizado para verificar a possibilidade de uma única equação representar a relação hipsométrica para todos os tratamentos agrupados ou pares de tratamentos. O referido teste baseia-se na comparação entre a soma de quadrado dos resíduos em cada tratamento (modelo completo-MC) e a soma de quadrado da diferença do modelo ajustado para os dados agrupados com todos os tratamentos (modelo reduzido-MR). Conforme Regazzi (1996), as hipóteses do teste são formuladas da seguinte forma:

-Hipótese de nulidade (H0): Os modelos completos não diferem estatisticamente do modelo reduzido;

-Hipótese alternativa (H1): ao menos um dos modelos completos difere estatisticamente do modelo reduzido.

Assim, quando o valor da estatística $\mathrm{F}$ for maior ou igual ao seu valor tabelado, indica significância a 95\% de confiança e rejeita-se a hipótese $\mathrm{H} 0$, ou seja, não é possível utilizar uma única equação para estimar a relação hipsométrica em diferentes tratamentos (REGAZZI; SILVA, 2010; ARAÚJO et al., 2012; CAMPOS; LEITE, 2013).

Em todos os procedimentos estatísticos deste estudo, adotou-se um nível de $5 \%$ de significância, e as análises estatísticas e ajustes dos modelos foram realizados no ambiente R (R CORE TEAM, 2016).

\section{RESULTADOS}

\subsection{Estatísticas descritivas}

A análise de variância demonstrou que os tratamentos exercem efeito no $\mathrm{d}$, ht e hc das árvores, ao nível de $5 \%$ de significância. Os resultados do teste de Tukey, bem como demais estatísticas descritivas estão apresentadas na Tabela 3.

As estatísticas descritivas das variáveis dendrométricas $(\mathrm{d}$, ht e hc) dos diferentes tratamentos (Tabela 3) permitiram inferir que há um aumento do diâmetro médio nos maiores espaçamentos, sendo que a maior média foi observada para o tratamento (T3). Além disso, nota-se uma maior variabilidade da estrutura diamétrica nos tratamentos (T1 e T2), expressa pelo desvio padrão e coeficiente de variação.

A variável altura apresentou maior média para o (T4) o qual apresenta maior densidade. Além disso, observa-se uma maior variabilidade das alturas nos maiores espaçamentos, expressa pelo maior desvio padrão e coeficiente de variação.

Tabela 3. Estatística descritiva e teste de Tukey para o diâmetro (d), altura total (ht) e comercial (hc) observados para as árvores mensuradas

\begin{tabular}{|c|c|c|c|c|c|c|}
\hline Tratamentos & Variável & Mínimo & Média & Máximo & Desvio padrão & Coeficiente de variação (\%) \\
\hline $\mathrm{T} 1$ & \multirow{4}{*}{$\mathrm{d}(\mathrm{cm})$} & 5,30 & $13,99 \mathrm{bc}$ & 20,80 & 4,75 & 34,01 \\
\hline $\mathrm{T} 2$ & & 5,00 & $12,35 \mathrm{c}$ & 19,40 & 4,08 & 33,05 \\
\hline $\mathrm{T} 3$ & & 9,40 & $17,04 \mathrm{a}$ & 23,70 & 2,83 & 16,64 \\
\hline $\mathrm{T} 4$ & & 8,60 & $14,60 \mathrm{~b}$ & 18,60 & 2,49 & 17,12 \\
\hline $\mathrm{T} 1$ & \multirow{4}{*}{ ht (m) } & 6,80 & $16,43 \mathrm{~b}$ & 22,20 & 4,39 & 26,74 \\
\hline $\mathrm{T} 2$ & & 8,90 & $17,65 \mathrm{~b}$ & 22,50 & 3,38 & 19,20 \\
\hline $\mathrm{T} 3$ & & 14,40 & $19,65 \mathrm{a}$ & 23,20 & 2,06 & 10,48 \\
\hline $\mathrm{T} 4$ & & 15,20 & $19,78 \mathrm{a}$ & 23,30 & 2 & 10,14 \\
\hline $\mathrm{T} 1$ & \multirow{4}{*}{ hc (m) } & 2,35 & $12,31 \mathrm{~b}$ & 17,70 & 4,75 & 38,64 \\
\hline $\mathrm{T} 2$ & & 1,30 & $12,83 \mathrm{~b}$ & 19,13 & 4,51 & 35,15 \\
\hline $\mathrm{T} 3$ & & 13,3 & $18,80 \mathrm{a}$ & 22,3 & 2,11 & 11,25 \\
\hline $\mathrm{T} 4$ & & 14,30 & $18,92 \mathrm{a}$ & 22,30 & 1,9 & 10,56 \\
\hline
\end{tabular}
no sistema de integração Lavoura-Pecuária-Floresta.

Table 3. Descriptive statistics and Tukey test for diameter (d), total height (ht) and commercial (hc) observed for the trees measured in the croplivestock-forest integration system. 
3.2 Modelagem da altura

tratamento T3 o modelo mais acurado foi Curtis com $\mathrm{R}^{2}$ aj. de

Os coeficientes e estatísticas das equações ajustadas nos $77,711 \%$ e $\mathrm{S}_{\mathrm{yx}}$ de $7,06 \%$.

diferentes tratamentos estão na Tabela 4. O teste de significância Nos resultados referentes ao ajuste para estimativa da hc dos coeficientes para ht apresentou resultados significativos (Tabela 5), observou-se pelo teste de significância que todos para todos os tratamentos $(\mathrm{p} \leq 0,05)$, indicando que os mesmos os modelos apresentaram coeficientes significativos.

explicam a variabilidade da altura total em função do diâmetro.

O modelo mais acurado no tratamento T1 foi Henriksen

Dentre as equações, Assmann apresentou maior acurácia de ajuste para os tratamentos $\mathrm{T} 1, \mathrm{~T} 2$ e $\mathrm{T} 4$ com $\mathrm{R}^{2}$ aj. variando

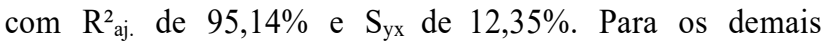
de $79,201 \%$ a $94,439 \%$ e $\mathrm{S}_{\mathrm{yx}}$ de $6,46 \%$ a $11,37 \%$. Para tratamentos o Hiperbólico foi o mais acurado com $\mathrm{R}^{2}$ aj. variando de 76,33 a $95,30 \%$ e $\mathrm{S}_{\mathrm{yx}}$ entre 6,92 e $7,90 \%$.

Tabela 4. Coeficientes e estatísticas dos modelos de relação hipsométrica ajustados para altura total nos diferentes tratamentos de integração Lavoura-Pecuária-Floresta.

Table 4. Coefficients and statistics of the adjusted hypsometric relation models for ht in the different treatments of integration-CroplandLivestock-Forest.

\begin{tabular}{|c|c|c|c|c|c|}
\hline Tratamento & Modelo & $\hat{\beta}_{0}$ & $\hat{\beta}_{1}$ & $\mathrm{R}^{2}$ aj. $(\%)$ & $\mathrm{S}_{\mathrm{yx}}(\%)$ \\
\hline \multirow{5}{*}{$\mathrm{T} 1$} & Stoffels & $0,8795^{*}$ & $0,7298^{*}$ & 86,605 & 14,20 \\
\hline & Curtis & $3,3965^{*}$ & $-7,6616^{*}$ & 90,310 & 12,08 \\
\hline & Hiperbólico & $20,1831^{*}$ & $-434,8450 *$ & 90,803 & 11,76 \\
\hline & Henricksen & $-7,981^{*}$ & $9,5070^{*}$ & 89,855 & 12,36 \\
\hline & Assmann & $24,6589 *$ & $-98,0210^{*}$ & 91,405 & 11,37 \\
\hline \multirow{5}{*}{$\mathrm{T} 2$} & Stoffels & $1,5138^{*}$ & $0,5447^{*}$ & 91,250 & 8,10 \\
\hline & Curtis & $3,3652 *$ & $-5,5915^{*}$ & 94,142 & 6,63 \\
\hline & Hiperbólico & $21,3610^{*}$ & $-378,0975^{*}$ & 92,770 & 7,37 \\
\hline & Henricksen & $-3,5445^{*}$ & $8,6408^{*}$ & 93,273 & 7,10 \\
\hline & Assmann & $25,6830 *$ & $-87,1496^{*}$ & 94,439 & 6,46 \\
\hline \multirow{5}{*}{$\mathrm{T} 3$} & Stoffels & $1,6507^{*}$ & $0,4685^{*}$ & 76,663 & 7,23 \\
\hline & Curtis & $3,4100 *$ & $-7,2361^{*}$ & 77,711 & 7,06 \\
\hline & Hiperbólico & $23,1814^{*}$ & $-931,83^{*}$ & 76,651 & 7,23 \\
\hline & Henricksen & $-4,819^{*}$ & $8,674^{*}$ & 77,316 & 7,13 \\
\hline & Assmann & $27,693 *$ & $-132,957^{*}$ & 77,675 & 7,07 \\
\hline \multirow{5}{*}{$\mathrm{T} 4$} & Stoffels & $1,8144^{*}$ & $0,4372 *$ & 78,277 & 6,74 \\
\hline & Curtis & $3,3914^{*}$ & $-5,8158^{*}$ & 79,080 & 6,62 \\
\hline & Hiperbólico & $23,3702 *$ & $-689,0461^{*}$ & 78,944 & 6,64 \\
\hline & Henricksen & $-2,3078^{*}$ & $8,2892 *$ & 78,709 & 6,68 \\
\hline & Assmann & $27,5547 *$ & $-109,7516^{*}$ & 79,201 & 6,60 \\
\hline
\end{tabular}

Tabela 5. Coeficientes e estatísticas dos modelos de relação hipsométrica ajustados para altura comercial nos diferentes tratamentos de integração Lavoura-Pecuária-Floresta.

Table 5. Coefficients and statistics of the adjusted hypsometric relation models for he in the different treatments of integration-CroplandLivestock-Forest.

\begin{tabular}{cccccc}
\hline Trat. & Mod. & $\hat{\beta}_{0}$ & $\hat{\beta}_{1}$ & $\mathrm{R}^{2}$ aj. (\%) & Syx (\%) \\
\hline \multirow{4}{*}{$\mathrm{T} 1$} & Stoffels & $-0,9318^{*}$ & $1,2955^{*}$ & 90,230 & 17,52 \\
& Curtis & $3,5283^{*}$ & $-13,5021^{*}$ & 94,667 & 12,94 \\
& Hiperbólico & $16,4419^{*}$ & $-478,7642^{*}$ & 92,216 & 15,71 \\
& Henricksen & $-15,8123^{*}$ & $10,9519^{*}$ & 95,146 & 12,35 \\
& Assmann & $21,5653^{*}$ & $-110,2488^{*}$ & 94,706 & 12,89 \\
\hline & Stoffels & $-0,7104^{*}$ & $1,2897^{*}$ & 85,955 & 18,81 \\
& Curtis & $3,7088^{*}$ & $-13,6231$ & 93,492 & 12,80 \\
& Hiperbólico & $17,9131^{*}$ & $-517,8851^{*}$ & 95,305 & 7,90 \\
& Henricksen & $-16,2953^{*}$ & $11,8741^{*}$ & 96,139 & 9,86 \\
& Assmann & $23,8520^{*}$ & $-119,5749^{*}$ & 97,234 & 8,34 \\
\hline & Stoffels & $1,5139^{*}$ & $0,5009^{*}$ & 75,835 & 7,90 \\
& Curtis & $3,3973^{*}$ & $-7,7730^{*}$ & 77,022 & 7,70 \\
& Hiperbólico & $22,4062^{*}$ & $-951,5615^{*}$ & 76,330 & 7.48 \\
& Henricksen & $-6,007^{*}$ & $8,794^{*}$ & 76,601 & 7,77 \\
& Assmann & $26,98^{*}$ & $-135,20^{*}$ & 77,114 & 7,69 \\
\hline & Stoffels & $1,7624^{*}$ & $0,4399^{*}$ & 76,103 & 7,37 \\
& Curtis & $3,3497^{*}$ & $-5,8612^{*}$ & 76,909 & 7,24 \\
& Hiperbólico & $22,3725^{*}$ & $-662,6270^{*}$ & 76,953 & 6,92 \\
& Henricksen & $-2,242^{*}$ & $7,942^{*}$ & 76,535 & 7,30 \\
& Assmann & $26,3804^{*}$ & $-105,3146^{*}$ & 77,074 & 7,22 \\
\hline
\end{tabular}

\footnotetext{
* significativo $(\mathrm{p} \leq 0,05)$
} 
Para uma melhor avaliação da qualidade dos ajustes, e com o intuito de verificar se as suposições da análise de regressão foram atendidas, foram gerados os gráficos de resíduos e da curva estimada versus observada para os modelos de maior acurácia para estimativa da ht e hc nos diferentes tratamentos (Figuras 1 e 2).

A distribuição gráfica dos resíduos apresentou-se homogênea e sem tendências ao longo da linha de regressão, indicando que as equações atenderam à pressuposição de independência dos resíduos.

As curvas hipsométricas representaram bem os dados observados e comportamento esperado para tal relação, com tendência estabilização do crescimento em altura com o acréscimo dos diâmetros.
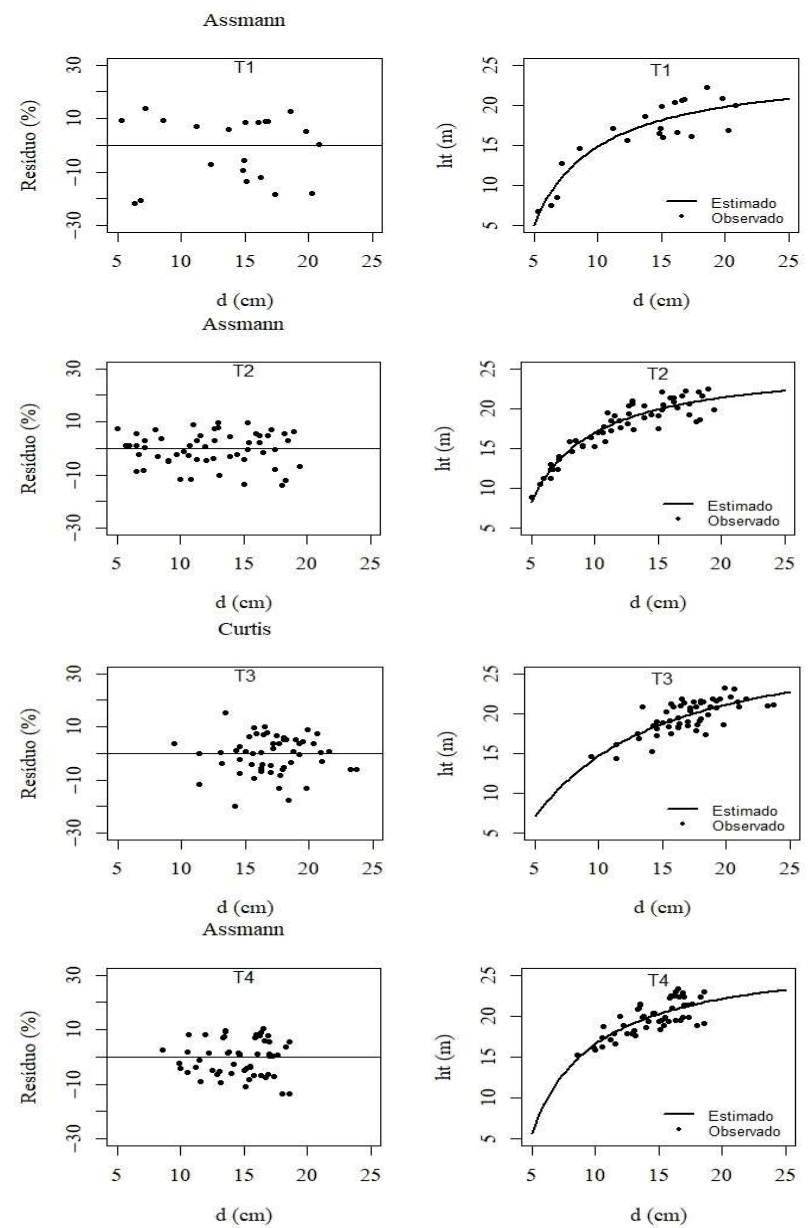

Figura 1. Distribuição dos resíduos e curva estimada versus dados observados para altura total nos diferentes tratamentos.

Figure 1. Distribution of residues and estimated curve versus observed data for ht in different treatments.

\subsection{Identidade de modelos}

$\mathrm{O}$ teste de identidade foi realizado com a equação que apresentou o resultado mais acurado na maioria dos tratamentos (Assmann). Apesar de um mesmo modelo ter sido selecionado para mais de um tratamento, observa-se por meio das curvas hipsométricas (h/d) que essa relação sofre influência dos diferentes tratamentos em função do espaçamento e arranjo de plantio. Essas diferenças podem ser visualizadas pela curva h/d gerada pela equação nos diferentes tratamentos (Figura 3).

Nota-se que a curva dessa relação é dinâmica, variando quanto á forma, inclinação e origem. As curvas da relação h/d foram mais íngremes nos tratamentos T4 e T3, suavizando ligeiramente para a direita nos tratamentos $\mathrm{T} 1$ e T2.
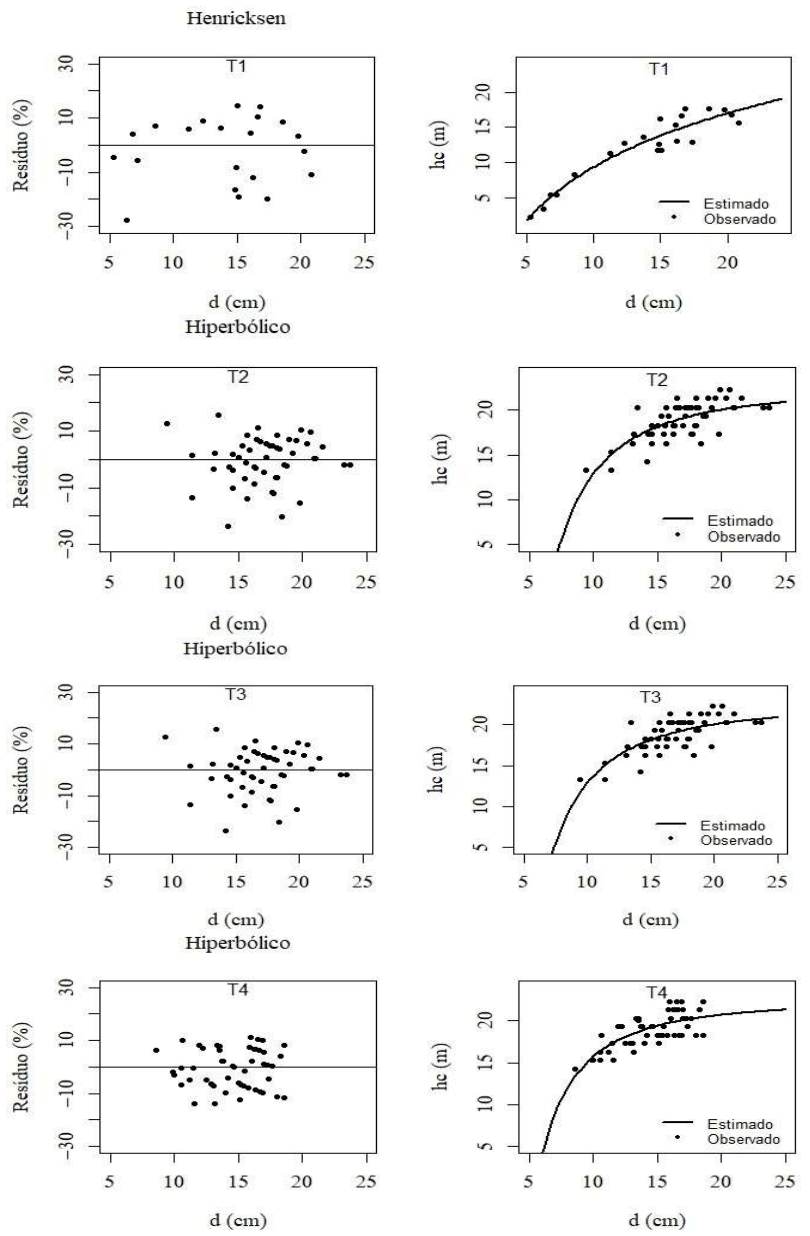

Figura 2. Distribuição dos resíduos e curva estimada versus dados observados para altura comercial nos diferentes tratamentos.

Figure 2. Distribution of residues and estimated curve versus observed data for hc in different treatments.
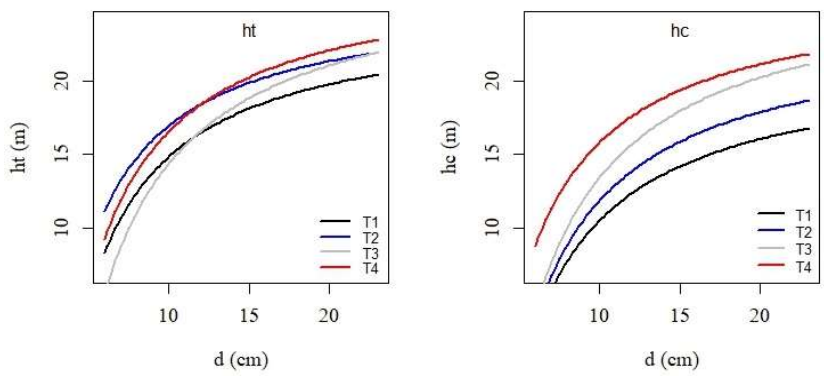

Figura 3. Curvas hipsométricas pelo modelo de Assmann para altura total (ht) e altura comercial (hc) nos diferentes tratamentos (T1, T2, $\mathrm{T} 3$ e T4).

Figure 3. Hypsometric curves by the Assmann model for ht and hc in the different treatments (T1, T2, T3 and T4).

O teste de identidade de modelos para altura total com os quatro tratamentos agrupados apresentou resultado significativo ( $\mathrm{p}$-valor $\leq 0,05)$. Resultado significativo também foi observado para altura comercial ( $p$-valor $\leq 0,05)$. Desta forma, rejeitou-se a hipótese de que não há diferença significativa entre as curvas estimadas, ou seja, pelo menos uma equação difere das demais. Logo, a soma de quadrado de resíduos para o ajuste com os diferentes tratamentos agrupados 
difere estatisticamente da soma de quadrados do resíduo do modelo ajustado para cada tratamento separado.

Esse resultado permite inferir que a relação $\mathrm{h} / \mathrm{d}$ da ht e hc altera-se de forma significativa entre os tratamentos, consequentemente, não é recomendado aplicar uma equação geral para um conjunto total de dados, que envolve diferentes espaçamentos e arranjos de plantio em sistemas iLPF. Sendo assim, foram conduzidos os testes aos pares de tratamentos para definição quais tratamentos diferem entre si quanto a relação h/d (Tabela 6).

Tabela 6. Resultado do teste de identidade de modelos para ht e hc nas diferentes combinações de tratamentos de integração LavouraPecuária-Floresta.

Table 6 . Results of the model identity test for ht and he in the different combinations of crop-livestock-forest integration treatments.

\begin{tabular}{ccccc}
\hline Variável & Tratamentos & T2 & T3 & T4 \\
\hline \multirow{3}{*}{ ht } & T1 & S & NS & S \\
& T2 & - & S & NS \\
& T3 & - & - & S \\
\hline \multirow{4}{*}{ hc } & Tratamentos & T2 & T3 & T4 \\
\cline { 2 - 5 } & T1 & $\mathrm{S}$ & $\mathrm{S}$ & $\mathrm{S}$ \\
& $\mathrm{T} 2$ & - & $\mathrm{S}$ & $\mathrm{S}$ \\
& $\mathrm{T} 3$ & - & - & $\mathrm{S}$
\end{tabular}

$\mathrm{S}=$ significativo; NS = não significativo a $95 \%$ de confiança.

Verificou-se para a variável ht que apenas nas combinações dos tratamentos ( $\mathrm{T} 1 \quad \mathrm{x}$ T3) e (T2 $\mathrm{x}$ T4) apresentaram resultados não significativos, indicando que uma mesma equação pode expressar a relação hipsométrica dos tratamentos agrupados, sendo que as demais combinações apresentaram-se significativas denotando que não é possível o agrupamentos dos demais tratamentos serem representados por uma única equação ajustada.

Nota-se que os pares de tratamentos que podem ser representados por uma única equação coincidem com os espaçamentos mais amplos (T1 x T3) e mais adensados (T2 x T4) por apresentarem curvas da relação h/d semelhantes. Enquanto nas combinações avaliadas entre os pares de tratamentos para hc, rejeitou-se a hipótese de nulidade $\mathrm{H} 0$ $(\mathrm{P}<0,05)$ para todos os tratamentos.

\section{DISCUSSÃO}

O diâmetro das árvores é bastante influenciado pela densidade de plantio. Bartoszeck et al. (2004), afirmam que povoamentos muito densos tendem, naturalmente, a apresentar árvores com diâmetros menores em comparação com árvores de povoamentos menos adensados, em que a concorrência por espaço é menor e o crescimento em diâmetro é mais acentuado.

Apesar de vários estudos revelam que a altura das árvores é pouco influenciada pela densidade (BARTOSZECK et al., 2004; LEITE et al., 2006; ARAÚJO et al., 2012), verifica-se que a maior densidade de plantio resulta em maior competição pelas árvores e consequentemente maior crescimento em altura, devido a uma tendência natural da planta buscar luz. Contudo, esse maior crescimento também pode estar relacionado ao fato do T4 ser um monocultivo. Medeiros et al. (2015) avaliando o crescimento de Tectona grandis L.F. em sistemas consorciados, verificaram que as plantas crescem mais no monocultivo devido ao fato de estarem livres de competição interespecífica.

Os indicadores estatísticos de acurácia obtidos pelas melhores equações se assemelham a outros estudos de relação hipsométrica para eucalipto (MIRANDA et al., 2014; SOUZA et al., 2016) e, portanto, são considerados satisfatórios na modelagem para estimativa da altura de floresta plantada, sobretudo quando os valores de $\mathrm{R}_{\text {aj. }}^{2}$ superam $90 \%$ e $\mathrm{S}_{\mathrm{yx}}$ são inferiores a $10 \%$.

Nota-se uma maior dispersão e tendência em subestimativa e superestimativa da variável ht e hc para o T1, o que corrobora com os piores indicadores estatísticos de precisão $\left(\mathrm{R}^{2}\right.$ aj. e $\left.\mathrm{S}_{\mathrm{yx}}\right)$ obtidos neste tratamento (T1).

Apesar das equações para ht e hc no T1 apresentarem tendência em subestimativa e superestimativa, Melo et al. (2017) afirmam que esses casos podem ocorrer na análise de regressão, sugerindo que o modelo pode ser usado para a relação hipsométrica sem prejuízo nas estimativas. Verifica-se que no menor espaçamento (T4) a relação hipsométrica foi mais íngreme devido à maior competição entre as árvores. A densidade afeta mais o crescimento em diâmetro do que em altura e consequentemente a curva $\mathrm{h} / \mathrm{d}$ se apresenta mais íngreme (BARTOSZECK et al., 2004).

No espaçamento mais amplo (T1) a curva da relação h/d apresentou-se mais suave deslocando sua origem ligeiramente para a direita. Isso se deve principalmente pela menor competição entre as árvores, resultando em menor relação h/d quando comparado com povoamentos mais adensados. Bartoszeck et al. (2004) afirmam que em povoamentos com alta competição, a relação $\mathrm{h} / \mathrm{d}$ é maior.

Resultados semelhantes sobre a influência do espaçamento na relação $\mathrm{h} / \mathrm{d}$ também foram observados por Morais Neto et al. (2012), na verificação da identidade de modelos hipsométricos em diversos arranjos de sistema agrossilvipastoril com eucalipto em Planaltina, DF. Araújo et al. (2012), testando modelos hipsométricos para Candeia (Eremanthus erythropappus) em diferentes espaçamentos de plantio em Minas Gerais, concluíram por meio do teste de identidade de modelos que não é possível aplicar uma única equação contemplando diferentes espaçamentos.

\section{CONCLUSÕES}

A equação de Assmann tem maior acurácia de ajuste para estimativa da altura total em sistemas iLPF com clones de Eucaliptus grandis $x$ Eucalyptus urophylla. Para estimativa da altura comercial o modelo Hiperbólico é o mais acurado.

$\mathrm{O}$ espaçamento e arranjo de plantio influenciam de forma significativa na relação hipsométrica, implicando que não se deve utilizar uma única equação para representar a relação $h / d$ para dados globais que contemplem diferentes espaçamentos e arranjo de plantio em sistemas iLPF.

Os resultados deste trabalho apontam a necessidade de desenvolver e aplicar equações específicas, quando envolve povoamentos com diferentes arranjos de plantio em sistemas de iLPF. Isso se torna insustentável devido a quantidade excessiva de amostragem de campo e equações. Desta forma, ao invés de continuar com o desenvolvimento de equações específicas, trabalhos futuros devem concentrar-se no desenvolvimento de equações gerais com o emprego da modelagem de efeitos mistos e técnicas de inteligência artificial, visando otimizar o gerenciamento de povoamentos florestais.

\section{AGRADECIMENTOS}

A Embrapa-Agrossilvipastoril; Ao Conselho Nacional de Desenvolvimento Científico e Tecnológico (CNPQ). 


\section{REFERÊNCIAS}

ARAÚJO, E. J. G.; PELISSARI, A. L.; DAVID, H. C.; SCOLFORO, J. R. S.; NETTO, S. P.; MORAIS, V. A. Relação hipsométrica para candeia (Eremanthus erythropappus) com diferentes espaçamentos de plantio em Minas Gerais, Brasil. Pesquisa Florestal Brasileira, Colombo, v. 32, n. 71, p. 257-268, 2012. DOI: https://dx.doi.org/10.4336/2012.pfb.32.71.257

ALVARES, C. A.; STAPE, J. L.; SENTELHAS, P. C.; GONÇALVES, J. L. M.; SPAROVEK, G. Köppen's climate classification map for Brazil. Meteorologische Zeitschrift, Berlin, v. 22, n. 6, p. 711-728, 2013. DOI: https://dx.doi.org/10.1127/0941-2948/2013/0507

BARTOSZECK, A. C. P. S. OLIVEIRA, E. B.; MACHADO, S. A. Modelagem da relação hipsométrica para bracatingais da região metropolitana de Curitiba-PR. Floresta, Curitiba, v. 32, n. 2, p. 189-204, 2002. DOI: https://dx.doi.org/10.5380/rf.v32i2

BARTOSZECK, A. C. P. S.; FIGUEIREDO FILHO, A.; OLIVEIRA, E. B.; MACHADO, S. A. Dinâmica da relação hipsométrica em função da idade, do sítio e da densidade inicial de povoamentos de bracatinga da Região Metropolitana de Curitiba, PR. Revista Árvore, Viçosa, v. 28, n. 4, p. 517-533, 2004. DOI: http://dx.doi.org/10.1590/S0100-67622004000400006

BINOTI, D. H. B.; BINOTI, M. L. M. S.; LEITE, H. G.; MELIDO, R. C. N.; SANTOS, F. L. Descrição da distribuição diamétrica de sistemas agrossilvipastoris utilizando as funções Weibull e hiperbólica. Revista Árvore, Viçosa, v. 36, n. 2, p. 349-356, 2012. DOI: https://dx.doi.org/10.1590/S0100-67622012000200016

CAMPOS, J. C. C.; LEITE, H. G. Mensuração florestal: perguntas e respostas. 4. ed. Viçosa: UFV, 2013. 605 p.

CURTIS, R. O. Height diamenter and height diamenter age equations for second growth Douglas-fir. Forest Science, Washington, v. 13, n. 4, p. 356-375, 1967.

GRAYBILL, F. A. Theory and application of the linear model. Belmont: Duxbury, 2000. 704p.

IBÁ INDÚSTRIA BRASILEIRA DE ÁRVORES. Relatório Anual 2016: Ano Base 2015. 2016. http://iba.org/images/shared/Biblioteca/IBA_RelatorioAn ual2016 .pdf. 28 dez. 2016.

LEITE, H. G.; NOGUEIRA, G. S.; MOREIRA, A. M. Efeito do espaçamento e da idade sobre variáveis de povoamentos de Pinus taeda L. Revista Árvore, Viçosa, v. 30, p. 603612, 2006. DOI: http://dx.doi.org/10.1590/S010067622006000400013

MACEDO, R. L. G.; VALE, A. B.; VENTURIN, N. Eucalipto em sistemas agroflorestais. 1. ed. Lavras: UFLA, 2010, $331 \mathrm{p}$

MACHADO, S. A.; FIGURA, M. A.; SILVA, L. C. R.; TÉO, S. J.; STOLLE, L.; URBANO, E. Modelagem volumétrica para bracatinga (Mimosa scabrella) em povoamentos da Região Metropolitana de Curitiba. Pesquisa Florestal Brasileira, Colombo, n. 56, p.17-29, 2008.

MEDEIROS, R. A.; PAIVA, H. N.; LEITE, H. G.; OLIVEIRA NETO, S. N.; VENDRÚSCOLO, D. G. S.; SILVA, F. T. Análise silvicultural e econômica de plantios clonais e seminais de Tectona grandis Lf em sistema taungya. Revista Árvore, Viçosa, v. 39, n. 5, p. 893-903, 2015. DOI:

67622015000500012
MELO, E. M.; CALEGARIO, N.; MENDONÇA, A. R.; POSSATO, E. L.; ALVES, J. A.; ISAAC JÚNIOR, M. A. Modelagem não linear da relação hipsométrica e do crescimento das árvores dominantes e codominantes de Eucalyptus sp. Ciência Florestal, Santa Maria, v. 27, n. 4, p. 1325-1338, 2017.2 DOI: http://dx.doi.org/10.5902/1980509829895

MENDONÇA, A. R.; CARVALHO, S. P. C.; CALEGARIO, N. Modelos hipsométricos generalizados mistos na predição da altura de Eucalyptus sp. Revista Cerne, Lavras, v. 21, n. 1, p. 107-115, 2015. DOI: https://dx.doi.org/10.1590/01047760201521011191

MIRANDA, R. O. V.; DAVID, H. C.; EBLING, A. A.; MÔRA, R.; FIORENTIN, L. D.; SOARES, I. D. Estratificação hipsométrica em classes de sítio e de altura total em plantios clonais de eucaliptos. Advances in Forestry Science, Cuiabá, v.1, n.4, p.113-119, 2014.

MORAES NETO, S. P.; PULROLNIK, K.; VILELA, L.; OLIVEIRA, P.; MARCHAO, R. L.; GUIMARAES JUNIOR, R.; MACIEL, G. A. Verificação da Identidade de Modelos Hipsométricos em diversos arranjos de sistema agrossilvipastoril. Planaltina: Embrapa Cerrado, 2012. 24 p. (Boletim de Pesquisa e Desenvolvimento, 310)

MÜllER, M. D.; SALlES, T. T.; PACIULlO, D. S. C.; BRIGHENTI, A.M.; CASTRO, C.R.T. Equações de altura, volume e afilamento para eucalipto e acácia estabelecidos em sistema silvipastoril. Floresta, Curitiba, PR, v. 44, n. 3, p. 473-484. 2014. DOI: http://dx.doi.org/10.5380/rf.v44i3.33149

R CORE TEAM. R: A language and environment for statistical computing. $\mathrm{R}$ Foundation for Statistical Computing, Vienna, Austria. 2006.

REGAZZI, A. J.; SILVA, C. H. O. Testes para verificar a igualdade de parâmetros e a identidade de modelos de regressão não-linear em dados de experimento com delineamento em blocos casualizados. Revista Ceres, Viçosa, v. 57, n. 3, p. 315-320, 2010.

REGAZZI, A. J. Teste para verificar a identidade de modelos de regressão. Pesquisa Agropecuária Brasileira, Brasília, v. 31, n. 1, p. 1-17, 1996.

SALLES, T. T.; LEITE, H. G.; OLIVEIRA NETO, S. N.; SOARES, C. P. B.; PAIVA, H. N.; SANTOS, F. L. Modelo de Clutter na modelagem de crescimento e produção de eucalipto em sistemas de integração lavourapecuária-floresta. Pesquisa Agropecuária Brasileira, Brasília, v. 48, n.2, p. 253 - 260, 2012.

SOUZA, H. S.; TSUKAMOTO FILHO, A. A.; VENDRUSCOLO, D. G. S.; CHAVES, A. G. S.; MOTTA, A. S. Modelos Hipsométricos para Eucalipto em Sistema de Integração Lavoura-Pecuária-Floresta. Nativa, Sinop, v. 4, p. 11-14, 2016. DOI: https://dx.doi.org/10.14583/2318-7670.v04n01a03

SCOLFORO, J. R. S. Biometria florestal. Lavras: UFLA/FAEPE, 2005. 352 p.

SCOLFORO, J. R. S. Biometria florestal: Modelos de crescimento e produção florestal. Lavras: UFLA, 2006. 393 p.

SEMA-MT_SECRETARIA DE ESTADO MEIO AMBIENTE DE MATO GROSSO. Características do Mato Grosso. 2014. Disponível em: $<$ http://www.sema.mt.gov.br/>. Acesso: 30/12/2017.

TORRES, C. M. M. E.; OLIVEIRA, A. C.; PEREIRA, BÁRBARA, L. C.; JACOVINE, L. A. G.; OLIVEIRA 
NETO, S. N.; CARNEIRO, A. C. O.; TORRES, C. M. M. E. Estimativas da produção e propriedades da madeira de eucalipto em Sistemas Agroflorestais. Scientia Forestalis, Piracicaba, v. 44, n. 109, p. 137-148, 2016. DOI: https://dx.doi.org/10.18671/scifor.v44n109.13

VENDRUSCOLO, D. G. S.; CHAVES, A. G. S.; SILVA, R. S.; SOUZA, H. S.; MEDEIROS, R. A.; MOTTA, A. S.; SILVA, F.T. Identidade em Modelos Hipsométricos para Tectona grandis com Diferentes Espaçamentos em Cáceres-MT. Nativa, Sinop, v. 3, n. 1, p. 44-49. 2015. DOI: http://dx.doi.org/10.14583/2318-7670.v03n01a07 